\title{
Imaging the orientation of myelin sheaths in a non-stained histological slice of human brain
}

\author{
Georg Schulz ${ }^{1,2}$, Hans Deyhle ${ }^{1,3,4}$, Christos Bikis ${ }^{1,5}$, Oliver Bunk ${ }^{3}$, and Bert Müller ${ }^{1 *}$ \\ ${ }^{I}$ Biomaterials Science Center, Dept. of Biomedical Engineering, University of Basel, Allschwil, Switzerland \\ ${ }^{2}$ Micro- and Nanotomography Core Facility, Dept. of Biomedical Engineering, University of Basel, Allschwil, \\ Switzerland \\ ${ }^{3}$ Paul Scherrer Institute, Villigen PSI, Switzerland \\ ${ }^{4}$ Diamond Light Source Ltd., Didcot, United Kingdom \\ Integrierte Psychiatrie Winterthur - Zürcher Unterland, Winterthur, Switzerland
}

Submitted: August 13, 2020

Accepted: September 10, 2020

Published: September 14, 2020

\section{Graphical Abstract}

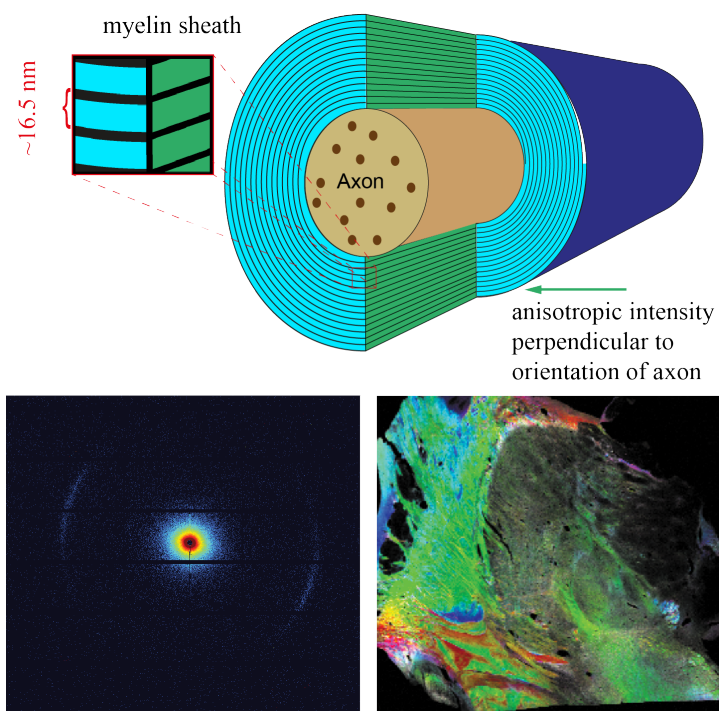

The periodical nanostructures of human brain tissues can be localized using spatially resolved small-angle X-ray scattering (SAXS). Prominent signals originate from myelin - as confirmed by histology. However, whereas histology requires suitable stains and only shows the abundance, spatially resolved SAXS also provides the color-coded orientation of myelin and related axons, and thus the characteristic tissue's anisotropy.

\section{Abstract}

The human brain is one of the most fascinating and important structures in nature. So far, histology has been the gold standard for imaging anatomical features on the sub-cellular level. Using standard optical microscopy spatial resolution is restricted to a fraction of a micron. Reciprocal-space techniques, including small-angle X-ray scattering (SAXS), with an inverse relationship between a nanostructure's size and scattering angle, have been used to study animal and human tissues. The myelin sheaths responsible for insulating axons, for example, exhibit a periodicity between 15 and $18 \mathrm{~nm}$. To localize their abundance and orientation, we have performed SAXS measurements with a micrometer-sized beam along a slice of the human brain and related them to the histology of myelin-stained tissue. The experimental results elucidate that, compared to histology, micrometer resolved SAXS provides information about the orientation of myelin, and consequently, axons, in addition to myelin abundance. The acquired data show color-coded orientation maps of the nanostructures, thereby providing a detailed insight into a relevant part of the human brain's nano-anatomy.

\section{Keywords:}

Human brain nanostructure, myelin sheath, synchrotron radiation, spatially resolved small-angle Xray scattering, histology, myelin staining

${ }^{*}$ Corresponding author: Biomaterials Science Center, Department of Biomedical Engineering, University of Basel, Allschwil, Switzerland, Phone: +41 6120754 30, E-mail: bert.mueller@unibas.ch 


\section{Purpose and Rationale}

For investigations of human brain structures on the cellular level and below, histology has been the gold standard. The histological slice's myelin staining provides information about the localization and abundance of myelinated nerve fibers in two preselected dimensions. In light microscopy spatial resolution is determined by the wavelength and, therefore, hardly better than one micrometer. This study is based on Xrays with a wavelength shorter than one nanometer, thus enabling the characterization of all kinds of nanometer-sized anatomical features. The combination of X-ray scattering and scanning allows for extracting average nanoscale data from the illuminated area and pinpointing their location on the micrometer scale. Within this study, we demonstrate that spatially resolved SAXS complements conventional imaging addressing the anisotropy characteristic for almost all human tissues. In this way, the orientation of axons, surrounded by the myelin sheaths, comes to light.

\section{Introduction}

A myelin sheath is a layer formed by myelin, which spirally surrounds the nerve axon, as illustrated in Fig. 1, and serves as an electrical insulator in order to speed up the conduction of neuronal impulses [1]. Such periodic nanostructures within the brain can be quantitatively investigated by so-called 'reciprocalspace techniques,' which are based on the inverse relationship between the size of the inspected nanostructures and the scattering angle. Schmitt et al. introduced X-ray diffraction (XRD) for the investigation of periodic nanostructures in animal nerves [2], and they noted four distinguishable spacings. Using small-angle X-ray scattering (SAXS), the periodicity of human myelin sheaths was determined at about $16.5 \mathrm{~nm}$ [3]. However, it is impossible to relate the SAXS results to established histology images due to the lack of localization. Combining SAXS with spatial resolution of a few micrometers in real space (scanning SAXS at cSAXS beamline, SLS, PSI, Switzerland [4]) provides vital information, including the nanostructure's abundance and orientation within the natural organization of the examined tissue [5]. Thus, these images give rise to a more detailed understanding of the brain's organization. Whereas diseases related to the abundance of myelin can be diagnosed post-mortem using conventional imaging and scanning SAXS, the latter can also be used to identify health deficits related to their orientation.

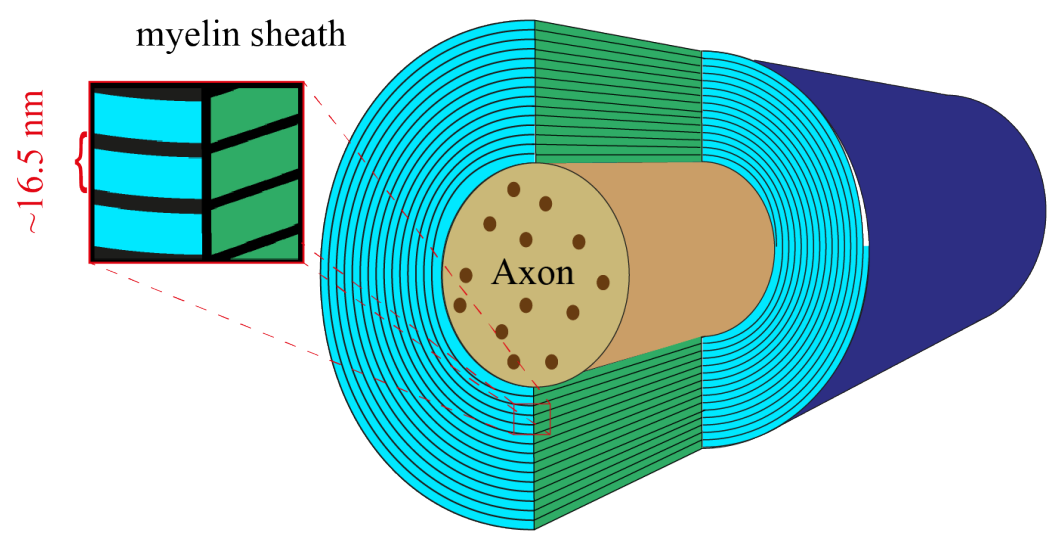

Figure 1: Appearance of myelin in brain tissues. The myelin sheath surrounds the axons. For an incident X-ray beam from the right side, anisotropic scattering intensity is expected.

\section{Materials and Methods}

\section{Brain tissue preparation}

For the present study, the brain was extracted from a donated body of an 87-year-old male with no neuropathological signs at autopsy. All procedures were conducted in accordance with the Declaration of Helsinki and according to the ethical guidelines of the Canton of Basel. One day after natural death, the brain was extracted at the Institute of Anatomy (University of Basel, Switzerland). The scalp was first detached, and then the calvaria was removed via a horizontal cut. After sectioning the tentorium cerebelli and a cut through the vasculature, nerves, and the medulla, the brain was extracted and put 
into $10 \%$ formalin for fixation. Before extraction and during the formalin fixation procedure, multiple magnetic resonance imaging (MRI) scans of the brain were performed to determine the influence of fixation on the inhomogeneous anisotropic brain tissue [6]. After two weeks of fixation, a block of the thalamus was obtained by the guillotine section at the University Hospital Zurich, Switzerland [7], and placed in a container filled with $4 \%$ formalin solution. The size of the thalamic block was $3 \times 3 \times 3 \mathrm{~cm}^{3}$.

Before thalamic block histology, X-ray grating-based phase-contrast microtomography of the specimen was performed in order to identify any deformations caused by cryo-sectioning and staining the histological slices [8]. After these experiments, the block was transferred into progressively increasing sucrose concentrations for approximately two weeks for cryoprotection. The block was then frozen by immersion in isopentane $\left(\sim-30^{\circ} \mathrm{C}\right)$ and stored at a temperature of $-75^{\circ} \mathrm{C}$. Using a Leica CM 3050, cryostat sections, $50 \mu \mathrm{m}$ thick, were collected in a $0.1 \mathrm{M}$ phosphate buffer [7]. For the staining procedure after the scanning SAXS experiments, the section was mounted on a gelatinized slide and myelin-stained using BlackGold II [9]. The section was then recorded (Canon EOS 40D, $60 \mathrm{~mm}$ close-up lens 1:2.8 EF-S).

\section{Scanning SAXS}

The scanning SAXS experiments were carried out at the cSAXS beamline at the Swiss Light Source, Paul Scherrer Institute, Villigen, Switzerland [4]. Using a photon energy of $11.2 \mathrm{keV}$, the beam was focused on a cross-section of $5 \times 20 \mu \mathrm{m}^{2}$ at the brain slice position for the raster scans. The brain section was placed in a poly-imide sachet with a phosphate buffer to avoid dehydration, and it was then scanned perpendicular to the beam in $100 \mu \mathrm{m}$ steps, i.e., in the $x$ - and $y$-directions. To acquire the scattering patterns, 24 PILATUS $2 \mathrm{M}$ detector modules [10], positioned at $7.096 \mathrm{~m}$ away from the specimen, were used. The slice was scanned with a 0.2 second exposure time per frame. The specimen-to-detector distance was determined using the first scattering order of a silver behenate calibration specimen. For the air-scattering reduction, an evacuated flight tube was placed between the specimen and the detection unit. Through such an experimental setup, data for an entire histology slice can be recorded within an hour.

\section{Data analysis}

To process the acquired data, a library established and available at the cSAXS beamline for MATLAB ${ }^{\circledR}$ was used. The fittings of the radially integrated signals, termed ' $q$-plots,' were performed by proFit 6.2.9 (QuantumSoft).
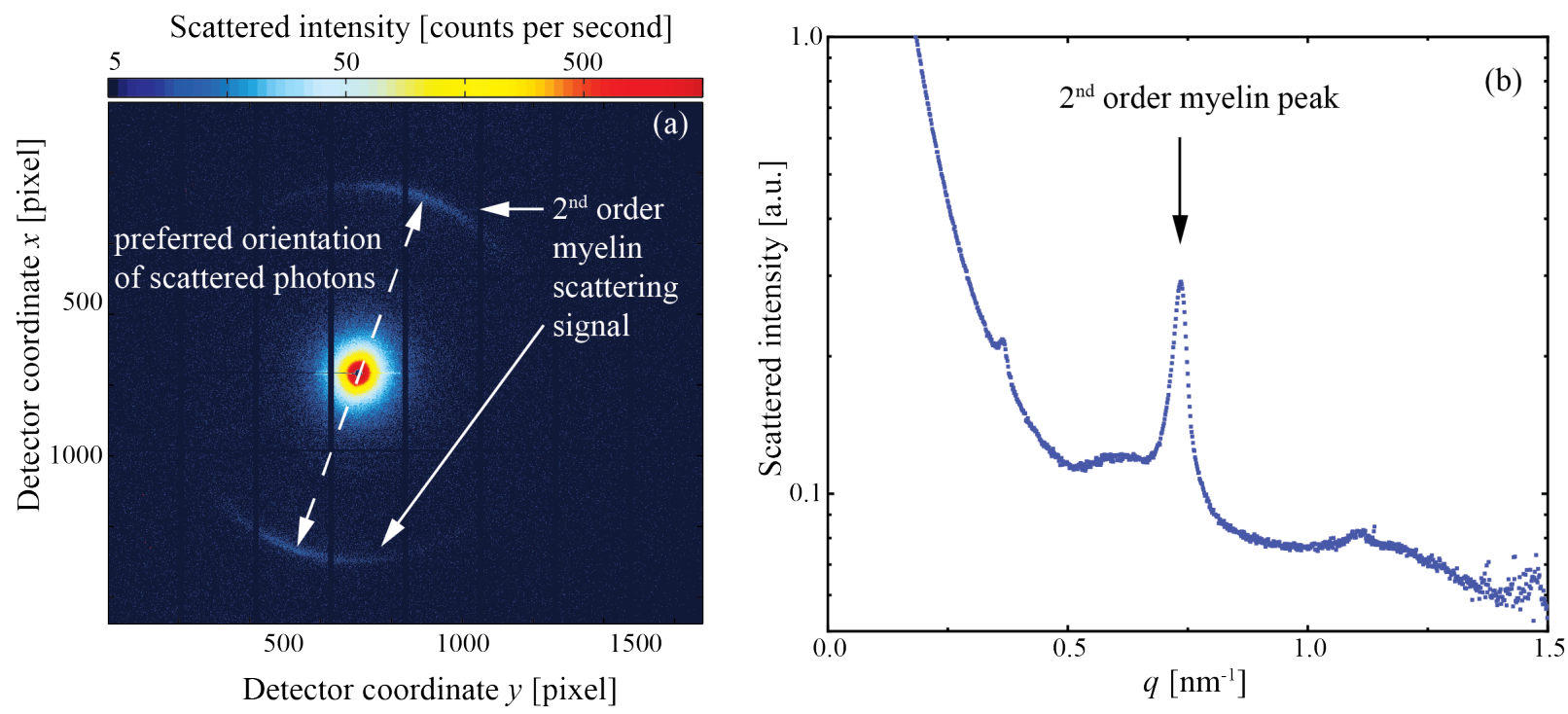

Figure 2: The SAXS pattern (a) of a selected position in the slice shows a clear signal with separated segments, which is characteristic of anisotropic nanostructures. The ring with the prominent segments corresponds to the second order of myelin sheath layers shown in the radially integrated intensity of the entire area analyzed (b). 


\section{Data registration}

To compare the scanning SAXS data directly with the corresponding myelin-stained histological slice, the two datasets were registered using the cross-correlation coefficient for matching [11]. Since local strain fields within the brain tissues occurred during histological sectioning $[8,12]$, an affine registration including three parameters for each translation, rotation, and scaling, would not provide adequate results. Therefore, a non-rigid registration, based on the adaptive hierarchical image subdivision strategy, which decomposes the nonrigid matching problem into numerous local affine registrations of sub-images of decreasing size, was applied $[11,13]$

\section{Results}

A characteristic scattering pattern of the thalamus slices illustrated in Fig. 2a exhibits a distinct ring with a well-defined scattered intensity orientation. This peak corresponds to the second-order myelin sheath's periodicity, and it is represented in the radially integrated q-plot (see Fig. 2. In addition to this characteristic peak, one finds two peaks and a shoulder. The first peak is associated with first order and the other one with third-order myelin sheath periodicity.

For a detailed investigation of the $q$-plot, zoom-ins of selected parts are combined into the diagrams in Fig. 3.

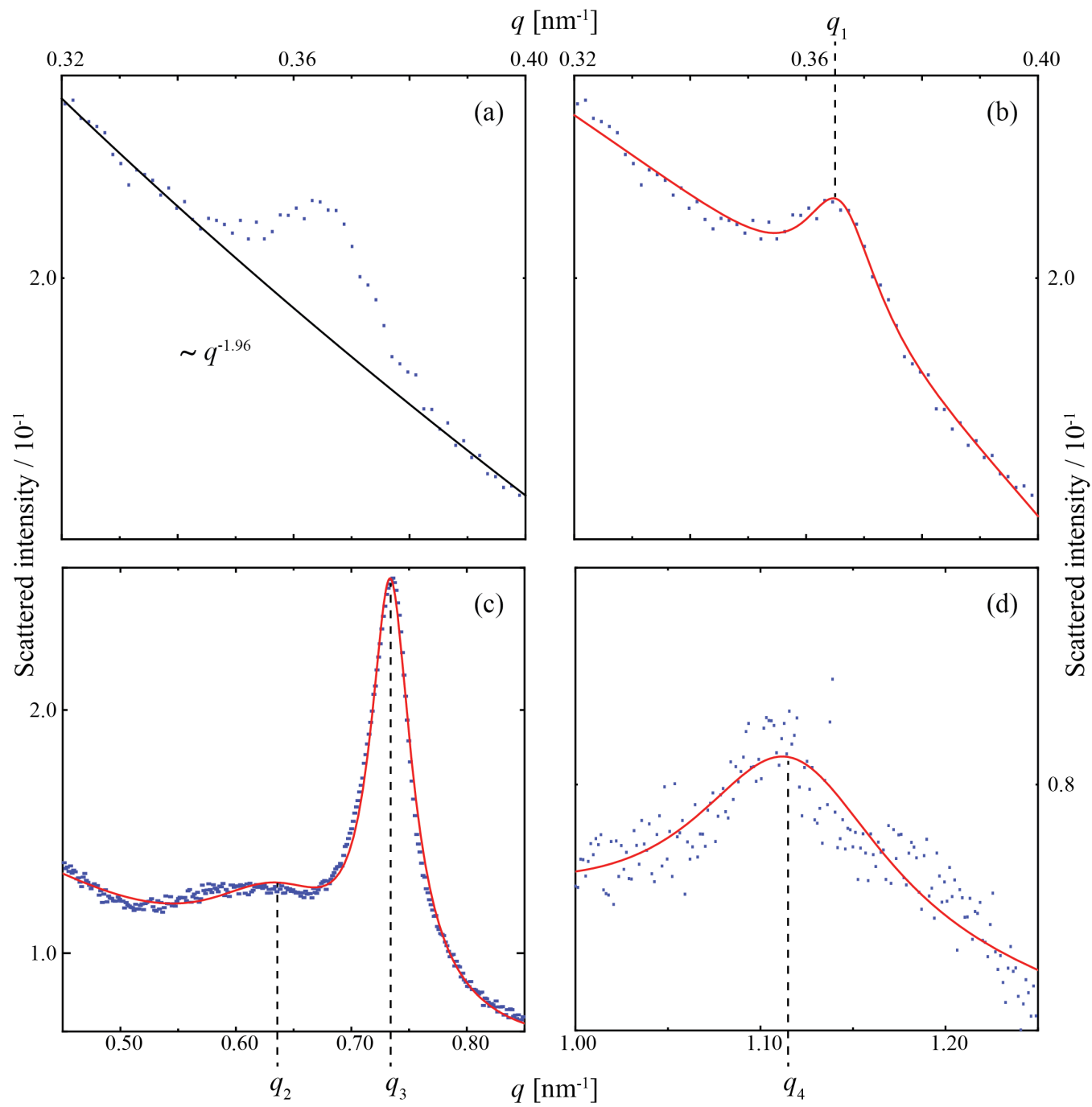

Figure 3: Radially integrated intensity for selected q-ranges. The peaks were extracted considering the background - cp. solid lines in diagram (a). The first- (b), second- (c) and third- (d) order myelin-related peaks, as well as a signal of unknown nanostructure marked by $q_{2}$, can be well approximated by means of Lorentzians with maxima at $q_{1}=(0.366 \pm 0.007) \mathrm{nm}^{-1}$, $q_{2}=(0.636 \pm 0.079) \mathrm{nm}^{-1}, q_{3}=(0.734 \pm 0.016) \mathrm{nm}^{-1}, q_{4}=(1.115 \pm 0.062) \mathrm{nm}^{-1}$, corresponding to real-space periodicities of $p_{1}=(17.17 \pm 0.12) n m, p_{2}=(9.88 \pm 0.78) n m, p_{3}=(8.56 \pm 0.14) n m$ and $p_{4}=(5.64 \pm 0.35) \mathrm{nm}$. 
To approximate base intensity, given by the background, which originated from air scattering, the polyimide sachet, the phosphate buffer, and the tissue itself, a power-law with an exponential decay of 1.96 was used (see Fig. 3 (a)).

According to the Porod law, the exponent is consistent with lamellar structures [14]. The intensity above the fitted curve is then related to the scattering signal from the nanostructures in the tissue. To determine the $q$-values - and thus the periodicities of the nanometer-sized anatomical features -, Lorentzian peaks with a
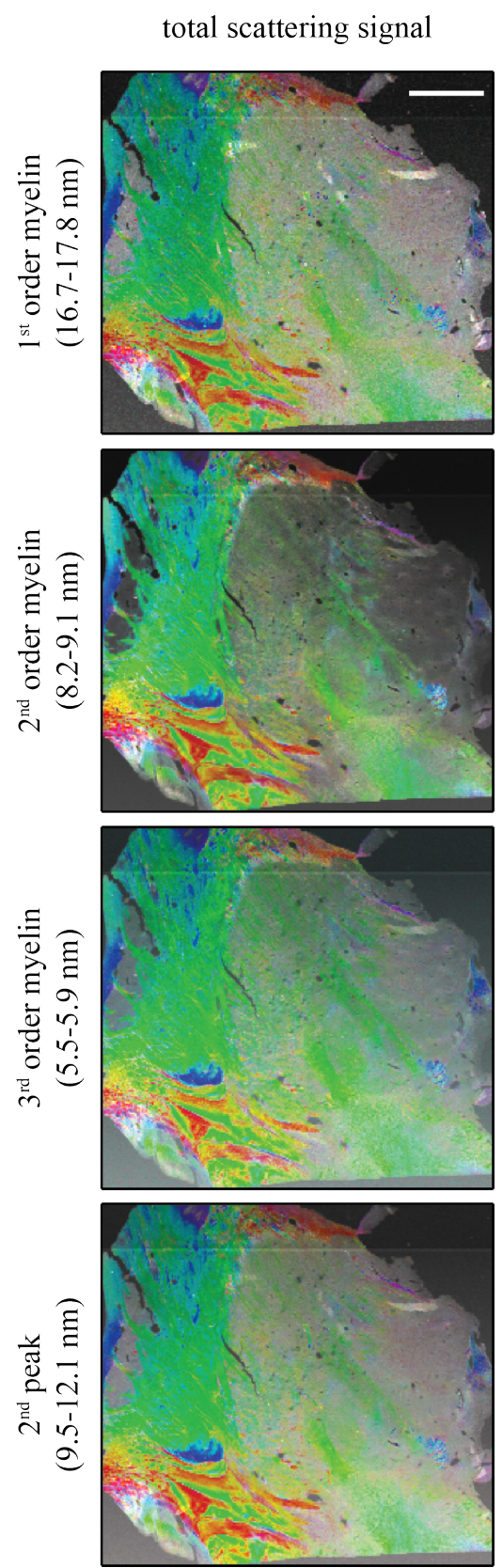

Figure 4: Processed scattering signals in the ranges of the four peaks. The orientation-dependent colors are according to the color disc, and their brightness is associated with nanostructure abundance. The sketch represents the orientation of the axons. The bar corresponds to a length of $5 \mathrm{~mm}$. first-order polynomial function were fitted to the three parts displayed in the diagrams in Fig. 3 (b-d). The mean values of the Lorentzians correspond to the $q$-values of the nanostructures. The first- (Fig. 3 (b)), second- (Fig. 3 (c) right peak), and third order (Fig. 3 (d)) peaks of myelin periodicity led to $(17.1 \pm 0.6) \mathrm{nm}$. The additional signal in the diagram in Fig. 3 (c) corresponds to a nanostructure with a periodicity of $(9.9 \pm 1.2) \mathrm{nm}$.

scattering signal after background subtraction
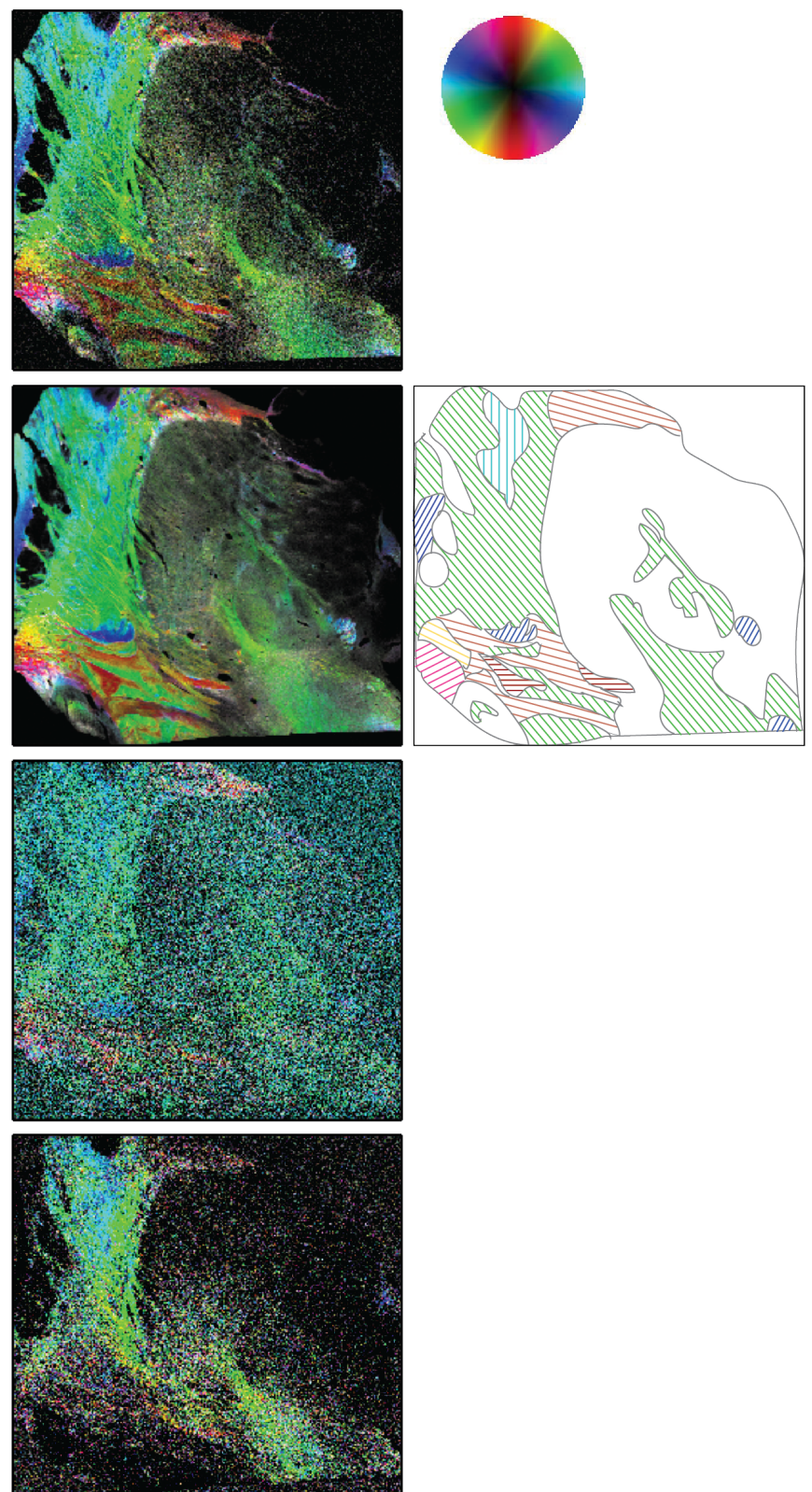

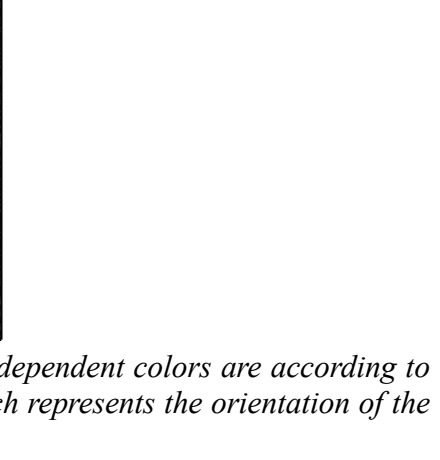


The color-coded images displayed in Fig. 4 are a combination of the abundance and orientation of the scattered photons for the $q$-values between $0.352 \mathrm{~nm}^{-1}$ and $0.376 \mathrm{~nm}^{-1}, 0.690 \mathrm{~nm}^{-1}$ and $0.766 \mathrm{~nm}^{-1}$ and $1.065 \mathrm{~nm}^{-1}$ and $1.142 \mathrm{~nm}^{-1}$, corresponding to the first- to third-order myelin-related signals, as well as between $0.519 \mathrm{~nm}^{-1}$ and $0.661 \mathrm{~nm}^{-1}$, corresponding to the unknown nanostructure's periodicity. The intensity of the scattered photons is represented by brightness, whereas the preferred orientation of the scattered signal, which was determined by approximating the azimuthal intensity distribution using a cosine [4], is according to the color wheel. Images in the first column show the measured signal, while images in the second column represent data after background elimination. Apart from the noise level, the images resemble each other. Myelin is mainly located around the thalamus - a hardly surprising fact.
Owing to the myelin sheaths' orientation, which spirally surround the nerve axons (see Fig. 1), the latter are oriented perpendicular to the scattering direction. This means that the main axon orientation, represented by the green color in the slice, is almost vertical. The unknown nanostructure labeled with $q_{2}$ is also mainly located outside the thalamus. Its abundance, however, differs from that of myelin; for example, in the region outside the thalamus, bottom-left the signal is almost absent.

To confirm some of the experimental findings, the slice was histochemically stained for myelin after the scanning SAXS experiment (see the image in Fig. 5 (a), and Fig. 5 (b) after conversion to grayscale and non-rigid registration to the SAXS image). The brightness of the histological slice directly relates to myelin concentration.

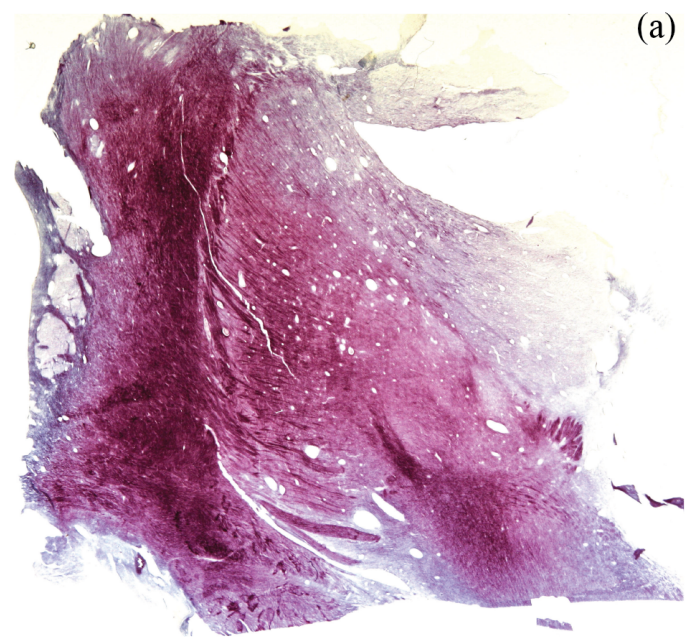

(a)
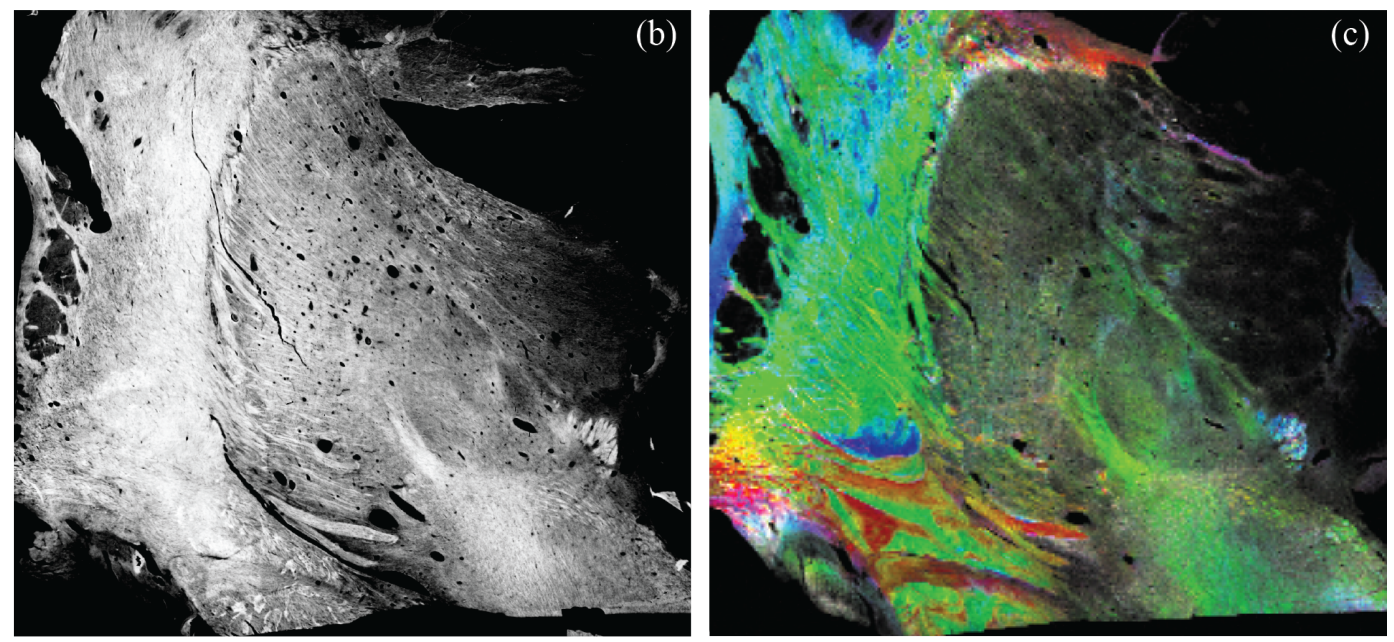

Figure 5: Myelin-stained slice (a), same slice after grayscale conversion and non-rigid registration, and the second-order myelin signal after background elimination (b) showing the abundance and orientation according to the color wheel of the myelin sheath. The bar corresponds to a length of $5 \mathrm{~mm}$. 
The common area of the two slices was found by non-rigid registration, in order to compensate for deformations caused by the staining procedure. The histological slice also proved that myelin is barely found inside the thalamus. A comparison between the histological slice and the second-order myelin-related spatially resolved SAXS data (see Fig. 5 (c)) verifies the local distribution of myelin in the selected brain tissue section.

\section{Discussion}

The scattering signal is proportional to the thickness of the tissue section. The upper limit is given by the X-ray absorption, which should be kept reasonably low. As a consequence, a previous study on human teeth [15] identified optimized thicknesses of a fraction of a millimeter. The present experiments, however, were based on histological brain sections, which are much thinner. Nonetheless, the signal-to-noise ratio was sufficient. Another issue is the role of the fixation and staining media. Staining is not necessary for the SAXS experiments, but the fixatives chemically modify the tissue components. Therefore, the choice of the fixation is essential for the measurements of myelin abundance and orientation. Consequently, synchrotron radiation-based spatially resolved SAXS of histology slices from soft tissues with thicknesses of a few micrometers is possible and less critical than conventional histology, because staining is avoided and thickness inhomogeneity can be corrected.

During previous myelin studies using SAXS, myelin's periodicity was analyzed, and a convincing explanation for the spacing given [3]. The present study is in perfect agreement with values more recently published $[16,17]$. The values for the spacing between myelin lamellae could depend on the tissue preparation - in the present study relied on formalin fixation, freezing, and cryo-sectioning. The reciprocal-space method provides only exact mean values about spacing and the abundance of periodic nanostructures within the illuminated area. Still, there is no way to determine their spatial distribution within the tissue. The scanning of a micrometer-sized beam enables the simultaneous localization of the nanostructures of interest within the macroscopic samples. Therefore, we can confirm that the three peaks of the $q$-plot belong to myelin, following a direct comparison with histology images collected after myelin-staining the thalamus slice. The bright regions in the histological slice, which indicate high myelin concentrations, correspond to high SAXS intensities.

There are two advantages of spatially resolved SAXS with respect to histology. First, staining is not necessary, and so any related tissue modifications are avoided. The second and main advantage of scanning SAXS in comparison to histology, however, is the possibility to evaluate the nanostructure's orientation quantitatively and thus to diagnose the anisotropy of the macroscopic parts of tissue on the nanometer scale.

The pixel size of the color-coded images shown in Figs. 4 and 5 are related to the step size in $x$ - and $y$-directions of the specimen, which was $100 \mu \mathrm{m}$ in the present study. The chosen step size leads to a scan time of around one hour for the thalamus slice with a size of $2.5 \times 2.5 \mathrm{~cm}^{2}$. A pixel size of $20 \mu \mathrm{m}$ would increase the acquisition time to one day (24 hours). The selected step size should correspond to the structural changes along the slice. The acquired images show extended regions with constant abundance and orientation. Thus, the step size was reasonably selected. For much more heterogeneous specimens, the limit is the focus size of the beam. Using X-ray lenses, this size can be further reduced by a factor of 20 , which leads to a spatial resolution comparable to optical microscopy and even beyond [18].The limitations of scanning SAXS originate from accessibility to synchrotron radiation facilities. In general, one has to apply for beamtime well in advance; therefore, only selected sections of a histological series can be taken into account for the scanning SAXS experiments before histochemical staining of all slices can take place. Using the scanning SAXS results of the selected sections, the orientation of myelin and related axons can be extrapolated to the entire histological study. For clinical purposes, including diagnostics of biopsies, the synchrotron radiation-based spatially resolved SAXS lacks accessibility. Laboratory-based setups, however, could certainly fit into the clinical environment. Spatially resolved measurements are still meaningless with these systems because of the low photon flux. Novel Xray sources (e.g., liquid metal source, Excillum, 
Sweden, or the Lyncean Compact Light Source) could be a valid tradeoff between flux and accessibility. The exact localization of the unknown nanostructures, namely the $q_{2}$-related signal, can support their identification. It might not be an additional periodic nanostructure, but it could have been created by the tissue preparation procedures. In previous studies, the $q_{2-}$ signal was interpreted as the human central nervous system (CNS) myelin $[3,16]$. The images in Fig. 4, however, exhibit many areas where myelin has been found, and the $q_{2}$-signal was weak or even absent. Therefore, it is questionable that the nanostructures related to the unknown peak are directly correlated with the axon cytoskeletal elements as proposed earlier $[3,16]$ because the signal should be much more pronounced and fit better to the spatial intensity distribution of myelin-related signals. Another explanation for the peak is the existence of a hexagonal phase [19], which is associated with a diseased state.

Knowing the orientation of myelinated nerve fibers in a three-dimensional tissue segment can be beneficial in validating established imaging approaches and complementing standards in connectomics research. For example, scanning SAXS studies could validate the results of diffusion tensor imaging (DTI) [20, 21]. Usually, such validation takes place by histology [22]. This tedious approach requires automated alternatives that are hindered due to inhomogeneous microscope lighting and staining intensities [23]. Using scanning SAXS, these technical challenges can be mastered.

Compared to histology validation alternatives, scanning SAXS investigations of myelin have further advantages. For instance, polarized light microscopy can be employed to visualize the orientation of nerve fibers in rather thick sections. The method relies on the birefringence of nervous fibers, a property lost after paraffin embedding [24]. Given the widespread use of paraffin embedding and the availability of paraffin-embedded samples in pathology laboratories and clinics, a compatible method thus has obvious merits. SAXS has been proven to work on paraffin-embedded samples from very early on [3], and even though the process disrupts the lipid structure, the more robust protein elements of myelin that survive actually generate the necessary SAXS signal attributed to paraffin-embedded tissue.
Correlating spatially resolved SAXS and DTI data is beneficial for validation purposes and allows for a substantial cross-fertilization since the physical information and the spatial resolution are complementary. Most important, the ex-vivo SAXS yields one to two orders of magnitude better spatial resolution than the in-vivo approach in DTI. The combination of the methods with computational sciences will lead to a complete connectomics atlas of the brain in health and disease. The importance of a complementary method to DTI for mapping neuronal tracts of all sizes is clearly stressed in the literature (see, e.g., ref. [25]), which proves that there is an inherent limitation in the use of DTI alone for detailed neuronal track analysis. This limitation is unlikely to be overcome soon by some technical adaptation of computational improvement. A recent pilot study combining DTI and scanning SAXS tomography on mouse brain indicates that these techniques complement each other well and that three-dimensional scanning SAXS can be used to validate the orientation information retrieved via DTI, and vice versa [17].

An attractive candidate for investigating myelinated fiber orientation is the optical chiasm. It has already been argued that detailed, truly three-dimensional information about the exact trajectories of the crossing nerve fibers can be of significant advantage [26]. Such advanced information will elucidate the preferential vulnerability of the decussating nasal fibers compared to their non-decussating temporal counterparts, by clearly identifying specific anatomic variations. Combined with the hypothesis that the relative direction of a compressive force, for example, caused by a tumor, with respect to fiber orientation significantly affects fiber survival, the importance of a SAXS tomography approach [27] offering isotropic three-dimensional information is evident in brain tumor animal models for cancer treatments. The myelin fiber orientation may determine the survival rate after radiation therapy and surgery.

The investigation of regenerating nerves assisted by biologically absorbable scaffolds presents another significant opportunity for SAXS. Such scaffolds have been used for nerve regeneration with promising results, and they clearly show not only the regeneration of the nerve but also the recovery of function [28]. The bio- 
materials used in this regard include bio-absorbable ones, such as collagen, which has been successful in mouse models [29]. In this context, the SAXS method offers a double advantage because there are characteristic periodicities in myelin and collagen. The opportunity to track the orientation of scaffold and regenerating nerves will support the optimization of related therapies. Also, one can determine to what

\section{Conclusions}

Using an X-ray beam of about $5 \mu \mathrm{m} \times 20 \mu \mathrm{m}$, we obtained strong myelin-related scattering signals from an unstained human brain slice several tens of micrometer thin. Shifting the slice perpendicular to the beam, two-dimensional images similar to histology were acquired. These images are complementary to histology data because they give not only access to the abundance of myelin but also to the orientation of the myelin sheaths. Consequently, the orientation of the axons was identified for several centimeter-squared-sized human brain slices. This approach could be readily extended to slices of the entire human brain in health and disease and does not rely on any staining protocol.

\section{Acknowledgments}

The authors gratefully acknowledge M. Müller-Gerbl, University of Basel, for the availability of the donated brain and the extraction of the brain, the technical support of A. Morel, University Hospital Zurich, for preparing the thalamus for cryo-sectioning, and of M. Imholz, University of Basel, for sectioning and staining the histological slice. The project was partially funded by the Swiss National Science Foundation, projects CR23I2_125406 "High-resolution 3D imaging of the human brain post-mortem" and CR23I2 147172 "Micro- and nanoanatomy of human brain tissues," and was supported by Swiss Light Source and the cSAXS beamline via proposal e12610. The authors thank G. Rodgers for proofreading the revision of the manuscript.

\section{Conflict of Interests}

The authors declare no conflicts of interest. For a signed statement, please contact the journal office: editor@precisionnanomedicine.com

Quote this article as Schulz G, Deyhle H, Bikis C, Bunk O, and Müller B, Imaging the orientation of myelin sheaths in a non-stained histological slice of human brain, Precis. Nanomed. 2020;3(3):656665, https://doi.org/10.33218/001c.17211

\section{References}

[1] M. Trepel, Neuroanatomie / Struktur und Funktion, 4 ed. Munich: Urban \& Fischer, 2008, p. 450.

[2] F. O. Schmitt, G. L. Clark, and J. N. Mrgudich, "X-ray diffraction studies on nerve," Science, vol. 80, pp. 567-568, 1934.

[3] M. De Felici, R. Felici, C. Ferrero, A. Tartari, M. Gambaccini, and S. Finet, "Structural characterization of the human cerebral myelin sheath by small angle x-ray scattering," Phys Med Biol, vol. 53, pp. 5675-5688, 2008.

[4] O. Bunk et al., "Multimodal x-ray scatter imaging," New J Phys, vol. 11, p. 123016, 2009.

[5] B. Müller et al., "Scanning X-ray scattering: Evaluating the nanostructure of human tissues," Eur J Clin Nanomed, vol. 3, pp. 30-33, 2010.

[6] G. Schulz, H. J. Crooijmans, M. Germann, K. Scheffler, M. Müller-Gerbl, and B. Müller, "Threedimensional strain fields in human brain resulting from formalin fixation," J Neurosci Methods, vol. 202, pp. 17-27, 2011.

[7] A. Morel, Stereotactic atlas of the human thalamus and basal ganglia. New York: Informa Healthcare 2007, p. 149.

[8] G. Schulz et al., "Evaluating the microstructure of human brain tissues using synchrotron radiationbased micro computed tomography," Proc SPIE, vol. 7804, p. 78040F, 2010. 
[9] L. Schmued and W. J. Slikker, "Black-Gold: a simple, high-resolution histochemical label for normal and pathological myelin in brain tissue sections," Brain Res, vol. 837, pp. 289-297, 1999.

[10] P. Kraft, A. Bergamaschi, C. Broennimann, R. Dinapoli, E. F. Eikenberry, and B. Henrich, "Performance of single-photon-counting PILATUS detector modules," J Synchrotron Radiat, vol. 16, pp. 368-375, 2009.

[11] A. Andronache, M. von Siebenthal, G. Székely, and P. Cattin, "Non-rigid registration of multimodal images using both mutual information and cross-correlation," Med Image Anal, vol. 12, pp. 3-15, 2008.

[12] M. Germann, A. Morel, F. Beckmann, A. Andronache, D. Jeanmonod, and B. Müller, "Strain fields in histological slices of brain tissue determined by synchrotron radiation-based micro computed tomography," J Neurosci Methods, vol. 170, pp. 149-155, 2008.

[13] B. Müller et al., "Three-dimensional registration of tomography data for quantification in biomaterials science," Int J Mater Res, vol. 103, pp. 242-249, 2012.

[14] O. Glatter and O. Kratky, Small-angle X-ray Scattering. Academic Press, London, 1982.

[15] H. Deyhle, S. N. White, O. Bunk, F. Beckmann, and B. Müller, "Nanostructure of the carious tooth enamel lesion," Acta Biomater., vol. 10, no. 1, pp. 355-364, 2014.

[16] T. H. Jensen et al., "Molecular X-ray computed tomography of myelin in a rat brain," NeuroImage, vol. 57, pp. 124-129, 2011.

[17] M. Georgiadis et al., "Retrieving neuronal orientations using 3D scanning SAXS and comparison with diffusion MRI," NeuroImage, vol. 204, p. 116214, 2020.

[18] A. Khimchenko et al., "Hard X-ray nano-holotomography: Large-scale, label-free, three-dimensional neuroimaging beyond optical limit," Advanced Science vol. 5, no. 6, p. 1700694, 2018.

[19] R. Shaharabani et al., "Structural Transition in Myelin Membrane as Initiator of Multiple Sclerosis," J. Am. Chem. Soc., vol. 138, pp. 12159-12165, 2016.

[20] P. Douek, R. Turner, J. Pekar, N. Patronas, and D. Le Bihan, "MR color mapping of myelin fiber orientation," J. Comp. Assist. Tomogr., vol. 15, no. 6, pp. 923-929, 1991.

[21] J. Coremans, R. Luypaert, F. Verhelle, T. Stadnik, and M. Osteauz, "A method for myelin fiber orientation mapping using diffusion-weighted MR images," Magn. Reson. Imaging, vol. 12, pp. 443-454, 1994.

[22] M. D. Budde and J. Annese, "Quantification of anisotropy and fiber orientation in human brain histological sections," Front. Integr. Neurosci., vol. 7, no. 3, pp. 1-8, 2013.

[23] A. Nazaran et al., "Methodology for computing white matter nerve fiber orientation in human histological slices," J Neurosci Methods, vol. 261, pp. 75-84, 2016.

[24] H. Axer, G. Berks, and D. Graf V. Keyserlingk, "Visualization of Nerve Fiber Orientation in Gross Histological Sections of the Human Brain," Microsc Res Tech, vol. 51, pp. 481-492, 2000.

[25] C. Thomas et al., "Anatomical accuracy of brain connections derived from diffusion MRI tractography is inherently limited," PNAS, vol. 111, pp. 16574-16579, 2014.

[26] N. S. Jain et al., "Visualization of Nerve Fiber Orientations in the Human Optic Chiasm Using Photomicrographic Image Analysis," Invest Ophthalmol Vis Sci, vol. 56, pp. 6734-6739, 2015.

[27] M. Liebi et al., "Nanostructure surveys of macroscopic specimens by small-angle scattering tensor tomography," Nature, vol. 527, pp. 349-352, 2015.

[28] K. Pawar et al., "Biomaterial bridges enable regeneration and re-entry of corticospinal tract axons into the caudal spinal cord after SCI: Association with recovery of forelimb function," Biomaterials, vol. 65, pp. 1-12, 2015.

[29] H. Suzuki et al., "Artificial collagen-filament scaffold promotes axon regeneration and long tract reconstruction in a rat model of spinal cord transection," Med Mol Morphol, vol. 48, pp. 214-224, 2015.

[30] C. Liu, R. Pyne, J. Kim, N. T. Wright, S. Baek, and C. Chan, "The Impact of Prestretch Induced Surface Anisotropy on Axon Regeneration," Tissue Eng, vol. 22, no. 2, pp. 1-11, 2016. 